\title{
SENTIMIENTOS Y RACIONALIDAD EN ECONOMÍA. COMENTARIOS AL LIBRO DE JORGE IVÁN GONZÁLEZ*
}

José Félix Catañoa

$\int 1$ orge Iván González presenta un libro de eruditas notas y reflexiones salidas de su larga trayectoria docente y consejero económico, que incluye ante todo unas ideas sobre las hipótesis de racionalidad de los agentes en el pensamiento económico, junto a temas distintos pero conexos, como cierto diagnóstico de la situación de la teoría económica, su metodología, su epistemología; y los criterios de su validez social.

Su propósito principal es mostrarnos que los grandes economistas y pensadores sociales han ya abandonado la hipótesis imaginaria del homos economicus (en adelante $H E$ ), idea aparentemente usada por los economistas en algunos modelos fundamentales (llamadas "construcciones imaginarias"), como el modelo de equilibrio general de los mercados de Arrow-Debreu y los primeros modelos microeconómicos de mercados perfectos ( $\sin$ fallas de mercado).

González describe esta hipótesis del $H E$ como la idea tradicional de la teoría neoclásica de un sujeto desapasionado y egoísta que busca optimizar, mediante un cálculo costo-beneficio, las posibilidades de obtener la satisfacción del consumo o las ganancias empresariales

DOI: https://doi.org/10.18601/01245996.v21n41.11. Recepción: 23-102018, modificación final: 28-02-2019, aceptación: 14-05-2019. Sugerencia de citación: Cataño, J. F. (2019). Sentimientos y racionalidad en economía. Comentarios al libro de Jorge Iván González. Revista de Economía Institucional, 21(41), 261-274.

a Profesor de la Universidad Nacional de Colombia, Bogotá, Colombia, [jfcatanom@unal.edu.co]. 
bajo la restricción de presupuesto; la queja subyacente es que en la formación de esa conducta no existe algún criterio social ni emocional, como si el sujeto de la teoría solo se atuviera a sus fríos criterios egoístas sin ser influenciado por sus relaciones con los demás ni por los pálpitos de su corazón ${ }^{1}$. Pero a veces, González va más allá. En efecto, hace el siguiente comentario sobre el modelo central de la teoría económica moderna, el modelo Arrow-Debreu:

En el modelo de equilibrio general no cabe un sujeto pensante, con pasiones y creencias. El agente que escoge es la última pieza que le falta al rompecabezas. Se le puede llamar homo economicus racional, pero también podría ser una rata o un gato. Cualquiera de estos tres seres cumple con el requisito que exige el modelo (p. 170).

Así, cabe dudar de que los sujetos humanos en el modelo referido ni siquiera hagan cálculos (son seres instintivos y no $H E$ ) o preguntar si los animales también hacen cálculos.

Ahora bien, según González, retomando una idea tomada de Kenneth Arrow, este sujeto natural y mecánico parece estar justificado por la estructura del modelo:

$[\mathrm{L}]$ os modelos... ponen el énfasis en las condiciones contextuales (precios flexibles, información completa, libre entrada y salida, calidad conocida, etc.)... Frente a estas restricciones de naturaleza contextual, al sujeto no le queda otra alternativa que actuar de una manera racional... Sin estos elementos restrictivos no habrá homo economicus racional (p. 17).

Una consecuencia posible de lo anterior es que si los contextos (inventados o "realistas") son los que definen los comportamientos, toda idea diferente de comportamiento tiene como condición definir ese contexto diferente. "Cuando el contexto se flexibiliza se hace evidente el comportamiento irracional de los agentes” (p. 37), piensa González. Esto significa que para abandonar el $H E$ es necesario poner en escena otras estructuras económicas o sociales, donde los agentes serían "irracionales”, lo que quiere decir que es afectado por emociones y creencias, pasiones, o los "espíritus animales" de Keynes, donde estaríamos frente al agente "real" y no al agente "imaginario".

${ }^{1}$ González llega a plantear que "En un espacio en el que solamente existen precios y cantidades no caben emociones" (p. 17). También afirma: "La noción de homo economicus conlleva dos prejuicios. El primero es de naturaleza reduccionista, así que lo único que importaría es la decisión racional, por fuera de los sentimientos y creencias. De allí se llega que el sujeto es maximizador después de que hace un ejercicio de costo-beneficio, en un contexto de equilibrio en el que actúan las cantidades y los precios. E1 segundo prejuicio es la atribución de esta manera de pensar a todos los economistas" (p. 22). 
La exposición central de González nos cuenta que los mismos economistas, principalmente los de la corriente principal, han superado el prejuicio y abandonado la hipótesis del $H E$. "Ningún autor serio considera que los seres humanos actúan exclusivamente por la razón” (p. 71). "La teoría económica contemporánea debe reconocer que la razón es insuficiente para entender las dinámicas complejas de la decisión" (p. 47).

Según nuestro autor, los grandes economistas hacen parte de los "autores serios", y en su texto encontramos las pruebas eruditas contundentes que muestran que A. Smith, M. Friedman, J. M. Keynes, G. Becker, J. Hicks, G. Shackle, H. Simon, Hayeck, L. von Mises, R. Coase, A. Hirshman, los clásicos, los institucionalistas, D. Kahneman, L. Hurwicz, todos ellos, se alejan de la "racionalidad estrecha" en muchos de sus planteamientos y de sus investigaciones -desde modelos descriptivos de mercados específicos a modelos de política económica-, o cuando los investigadores sociales hablan de cuestiones estrictamente ajenas a las situaciones económicas, como la elección social, la organización de la salud y la educación, la organización de la empresa, las familias y la política. Es normal entonces que leyendo el texto de González salgamos convencidos de que afortunadamente los grandes teóricos de la economía se han acercado a otras ciencias sociales e incorporado una imagen más "realista" del agente en sus análisis económicos y sociales, y que la hipótesis del $H E$ hace parte de los conceptos relegados por el progreso científico de los economistas.

Entremezcladas con este contenido principal, González expresa algunas opiniones sobre la situación de la teoría económica, sus formas de validación y sobre algunos autores específicos que merecen algunos comentarios.

\section{COMENTARIOS}

\section{SOBRE EL ABANDONO DE LA HIPÓTESIS DEL HOMO ECONOMICUS}

En un interesante artículo sobre la racionalidad individual, Benetti y Cartelier (1994) plantean que la hipótesis de racionalidad en economía tiene dos aspectos. El primero, derivado de la metodología del individualismo metodológico, según el cual la sociedad no existe al principio del análisis sino que proviene de las decisiones de los agentes y, por tanto, es obligatorio definirlos como entidades a-sociales, como si pertenecieran a un mundo natural (aquí la referencia es la tesis de Hobbes sobre el origen lógico de la sociedad). Esta posición sostiene que la sociedad no es el principio de explicación sino los individuos, 
como "si la sociedad fuera el resultado del ensamblaje de un conjunto de ladrillos que se denominan individuos" (ibid., p. 20), y por tanto, los economistas neoclásicos (y aquí debemos agregar a Smith en su teoría de la génesis de la división del trabajo) sostendrían que los agentes se convierten en comerciantes porque son por naturaleza calculadores y racionales. E1 segundo aspecto es pensar "las reglas que siguen los individuos cuando entran efectivamente en relaciones mercantiles" (ibíd.); en este segundo caso, se considera al sujeto en el sistema de coordinación (económica) con los otros, lo que requiere haber definido de alguna manera la naturaleza de las relaciones mercantiles (sin confundirlas con cualquier interacción humana), plantear sus mediaciones (el equilibrio, el subastador o el dinero, p. ej.) y las restricciones de su acción (el presupuesto o la solvencia). En síntesis, se tienen dos reflexiones sobre el tipo de comportamiento de los agentes, la primera se refiere a la manera en que los individuos hacen elecciones de estrategias posibles; la segunda a la manera en que los agentes se relacionan económicamente con los otros, en una coordinación que utiliza normas o instituciones.

Los modelos centrales de equilibrio general contienen tratamientos de los dos aspectos señalados por los profesores franceses. En el primer aspecto utilizan la idea de que los individuos pueden definirse como seres naturales dispuestos a conservar su autarquía o a tener relaciones de intercambio de bienes con otros, siempre y cuando en cada situación resulte una situación que mejore su función de utilidad material mediante el consumo de bienes físicos. Es por este lado que en principio aparece la noción de $H E$, como hipótesis inicial del análisis económico tradicional; así se muestra que bajo ciertas condiciones se obtienen dos resultados positivos y uno de carácter negativo. Primero, los positivos: 1) existe un equilibrio general, un estado social de compatibilidad de las decisiones individuales, 2) este equilibrio puede ser óptimo para los individuos (óptimo de Pareto). E1 negativo: 3) este modelo de individuos racionales no posee estabilidad ni unicidad, es decir, el equilibrio de los mercados es independiente del proceso coordinación mercantil, y una economía Arrow-Debreu puede poseer varias situaciones de equilibrio.

En cuanto al segundo aspecto -la coordinación mercantil-, el modelo trata el funcionamiento de los agentes en desequilibrio y en equilibrio, situaciones donde es necesario señalar cómo toman decisiones en el proceso de relacionarse unos con otros. Aquí encontramos, primero, que en el modelo Arrow-Debreu se introduce la poderosa figura del subastador que transmite la información de precios a los 
agentes, recibe sus respuestas y anuncia el momento del equilibrio, poderes por los que, además de los agentes, se introduce cierto tipo de mediaciones para modelar cómo se coordinan unos con otros antes de llegar al equilibrio, si es que lo logran. Un examen atento señala que en este proceso los agentes van a actuar irracionalmente, pues hacen propuestas virtuales a los precios dados por el subastador $\mathrm{y}$, a pesar de que en el momento siguiente los precios cambian, siguen creyendo que los precios que se les dan son de equilibrio y, por ende, no especulan ni engañan al poderoso deux ex machina; además, a pesar de que a ciertos precios sería ventajoso hacer transacciones, estos deseos son irrealizables porque la regla del juego prohíbe transacciones por fuera del equilibrio. Vemos entonces que los individuos por fuera del equilibrio, en el proceso de coordinación, se comportan de manera irracional, violando allí mismo la hipótesis del $H E$. Segundo, cuando los individuos saben que están en equilibrio, las transacciones son aparentemente viables siempre y cuando se añada al modelo una hipótesis de la técnica de transacciones (la caja de compensación de cuentas de Debreu, el trueque o el dinero) y esta mediación no se explica aplicando el criterio del $H E$, como demuestra el fracaso de la micro fundamentación del dinero en la teoría de los neo-walrasianos. Dadas estas características, algunos observadores han visto que el modelo Arrow-Debreu representa un mundo centralizado y autocrático y no un mundo abstracto de comerciantes libres, a pesar que se comporten de acuerdo con la hipótesis del $H E^{2}$.

Es con estos elementos de referencia que me permito examinar las posiciones de González. Como es tradicional entre economistas, su exposición principal se refiere al primer aspecto y no trata el segundo, aunque sí lo reconoce marginalmente ${ }^{3}$. Sin referirse al individualismo metodológico, considera el modelo Arrow-Debreu como arquetipo del uso de la hipótesis del $H E$ y a ella le atribuye grandes resultados: esta "maravillosa construcción imaginaria (tiene, además del óptimo), un equilibrio único y estable" (p. 26). También cita a Arrow cuando advierte que "el supuesto de racionalidad no es una condición de la teoría económica" (p. 38). De ser ciertas, estas dos declaraciones son evidentemente contradictorias.

${ }^{2}$ Ver Rebeyrol (1999).

${ }^{3}$ La única ocasión es cuando cita a Mantzavinos, North y Sharin cuando plantean que "un individuo que comienza a intercambiar en el mercado ya comparte reglas sociales con los otros participantes. No es un ser a-histórico que únicamente esté equipado con sus preferencias y que maximiza la utilidad bajo restricciones como, los precios y el ingreso disponible” (p. 77). 
En verdad, en Arrow no hay contradicción sino un mal entendido de González. Lo que sabe Arrow, y muchos economistas teóricos, es que la figura del agente maximizador no siempre es necesaria en el modelo Arrow-Debreu y que, al contrario de lo que afirma González, este modelo carece de unicidad y de estabilidad. El único resultado positivo del modelo neo-walrasiano es la existencia de equilibrios, unos óptimos y otros no. Se sabe también que para la existencia del equilibrio no es necesaria la hipótesis del agente maximizador, dado que esa existencia solo requiere, además de las hipótesis básicas (bienes, información y competencia perfecta, tiempo limitado, etc.), que la función $Z$ (un agregado de la multitud de disposiciones de compra y venta de los individuos sobre cada producto) de exceso de demanda sea continua, homogénea de grado cero y que cumpla la ley de Walras. Esto significa que es posible conseguir el equilibrio con funciones de exceso de demanda $Z$ (un agregado) donde el comportamiento de los agentes esté alejado del comportamiento maximizador. En otras palabras, el resultado colectivo no depende de cómo se comportan individualmente los agentes, sino de la forma que toman los agregados. Como dicen Durán y Lozano (2018) “dos economías Arrow-Debreu distintas pueden tener la misma función de exceso de demanda agregada, lo cual implica que no es posible caracterizar una economía Arrow-Debreu a partir de su función de exceso de demanda" (p. 269). Esto significa que el modelo central de la teoría económica contradice la idea de González de que es al arquetipo del uso de la hipótesis $H E$ (asimilada a la de la maximización), porque permite varios tipos de racionalidad ${ }^{4}$.

Lo que confunde a González (y no es al único) es que la hipótesis del $H E$ solo es esencial para probar que un equilibrio económico es un óptimo de Pareto, el primer teorema del bienestar; es decir, que es obligatoria para el modelo normativo (pero no para el modelo descriptivo), aquel que sirve de referencia para escoger el mundo ideal

${ }^{4}$ Aunque mantengamos la hipótesis del HE, la formación de la visión sobre lo que le interesa es un asunto privado del sujeto y no excluye las pasiones o las emociones. Si algunos sujetos escogen una película de terror en su función de utilidad y otros una película de Berman, es que tienen emociones diferentes. Además, la idea de que el $H E$ se puede asociar a una respuesta instintiva de los agentes es negar, no tanto la racionalidad estrecha de los agentes naturales, sino un sujeto que hace cálculos. González advierte desde la primera página que los comentaristas no eran rigurosos puesto que "la hipótesis de racionalidad está llena de lugares comunes, y que no hay una reflexión sistemática sobre el sentido de las categorías” (p. 15). Aquí vemos que no cumple su propia exigencia, por lo menos en este caso, una base central de su argumentación general.

Revista de Economía Institucional, vol. 2i, N. ${ }^{\circ}$ 4i, Segundo Semestre/2oi 9, pp. 26 i-274 ISSN OI $24-5996 / \mathrm{E}-$ ISSN $2346-245^{\circ}$ 
deseado como para las construcciones teóricas que dan cuenta de mundos sub-óptimos, los célebres modelos con "fallas del mercado". En general, la teoría neoclásica del equilibrio general dirá que un mundo donde operen racionalidades no maximizadoras podrá tener un equilibrio sub-óptimo siempre y cuando su agregado cumpla las condiciones antes anunciadas. Esta es la razón para que Arrow haya dicho que esta hipótesis no es necesaria para la teoría económica... si es una ciencia descriptiva de la economía, preocupada por el "ser" y no por el "deber ser"

Ahora bien, puesto que la existencia del equilibrio general no depende de la $H E$, no es una gran sorpresa que los economistas hayan incorporado otras versiones del comportamiento individual sin tener que modificar el equilibrio como idea básica de la teoría ni declarar muerto o inútil el modelo Arrow-Debreu 6 . Esto explica porqué la teoría de la corriente principal se ha construido sobre el binomio comportamiento individual y equilibrio, y que la modificación de la idea que se tenga sobre los agentes no parece haber modificado la idea de equilibrio ni la del mercado, pues lo que está en juego al cambiar la racionalidad de los agentes es tan solo la calidad del equilibrio posible. Aún más, la importancia de la hipótesis de la racionalidad individual ha aumentado porque algunos modelos macroeconómicos y microeconómicos adoptan la hipótesis de que los agentes son iguales entre sí y, por tanto, los resultados "sociales" dependen, ahora sí directamente, no de la forma del agregado, sino del comportamiento del agente "representativo". Parece confirmarse que mientras la sociedad más se reduce a un individuo (suprimiendo el proceso de relación entre individuos distintos), el principio de racionalidad es más importante para explicarla, pues si se conoce la función de comportamiento del agente representativo, se conocerán los resultados, como saben los economistas para el caso del solitario Robinson Crusoe. En resumen, cuanto más se elude la relación con

${ }^{5}$ Para los neo ricardianos es algo similar. El equilibrio de precios de Sraffa no depende de alguna hipótesis de racionalidad. ¿Sraffa y Arrow están de acuerdo?

${ }^{6}$ González protesta porque la denominación de neoclásicos se atribuya a intelectuales como Hicks, Arrow o Samuelson sabiendo que ellos han aceptado que es necesario separarse de la hipótesis de $H E$. Esto es sintomático de su creencia de que la perspectiva neoclásica depende la hipótesis $H E$, como si salir de esa hipótesis nos alejara del paradigma neoclásico. Ya los protestantes le demostraron a los católicos que la hipótesis de la virginidad de María (algo no esencial para la doctrina) no era necesaria para declararse cristianos. 
los otros (un modelo de equilibrio o interdependencia general) más trascendental es la hipótesis de racionalidad.

Aparte del error anterior, la exposición de González es importante porque muestra que si bien fueron los economistas de la corriente principal quienes introdujeron la figura radical del $H E$ (no aparecía así ni en Smith ni en los clásicos), ellos mismos son los que se han alejado de ella en el análisis de diversos problemas económicos y sociales. Su conclusión es ahora generalmente compartida, como indican textos de dos recientes premios Nobel. Primero, Thaler, quien escribió:

Sin embargo, debe dejarse de creer que estos modelos con Econos (los agentes $H E$ ) dan una descripción exacta del comportamiento humano y dejar de tomar las decisiones de política económica sobre la base de análisis también erróneos. Es el momento de tomar en cuenta los factores supuestamente irrelevantes... Durante años, muchos economistas han fuertemente resistido a las exhortaciones para que se basen sus modelos en una descripción más justa del comportamiento humano. Sin embargo, con la llegada de jóvenes economistas creativos que no han dudado en asumir riesgos y salirse de la manera tradicional de hacer el sueño de una teoría económica enriquecida se está llevando a cabo. Este nuevo campo se conoce como la "economía del comportamiento". No se trata de una nueva disciplina: es aún de la teoría económica, pero en la cual se han incorporado dosis de psicología y de varias otras ciencias sociales... La principal razón de por qué es necesario integrar a los seres humanos dentro de las teorías económicas, es que se debe mejorar la precisión de las predicciones hechas en el contexto de estas teorías. Pero tomar en cuenta la "gente real" tiene otra ventaja: la "economía del comportamiento" es mucho más interesante y más divertida que la economía convencional. Es lo opuesto a una ciencia triste (Le Monde, 5 de octubre, 2018).

Por su parte, Jean Tirole escribe:

E1 cuestionamiento del homo economicus ha empujado gradualmente a la economía a estar más cerca de otras ciencias sociales, para entender mejor el comportamiento humano y mejorar sus conocimientos, sus modelos y sus teorías. De esta manera, los economistas han aprendido mucho y el número de teorías económicas directamente influenciadas por una o más ciencias sociales está creciendo rápidamente desde la década de 1980. Esta tendencia ya se refleja en el otorgamiento de tres Nobel economía relacionado con la conducta (Daniel Kahneman en 2002, Robert Shiller en 2013 y Richard Thaler en 2017) y ciencias políticas (Elinor Ostrom en 2009). El homo economicus ha muerto, sustituido por un ser humano más complejo, más incierto, más difícil de comprender pero también más realista. Entre las numerosas contribuciones de las ciencias sociales a la economía se debe incluir la incorporación del homo socialis. Los sociólogos insisten, con razón, en la importancia de dejar de analizar el individuo fuera de contexto, es decir, sin considerar su ambiente social. E1 individuo es parte de estos grupos y estos afectan de múltiples maneras 
cómo se comporta el individuo: transmisión de cultura y creencias, presión social, búsqueda de identidad y pertenencia al grupo, confianza en los otros, influencia de la reputación de un grupo social sobre los individuos, narrativas que circulan en el seno de los grupos, estructuras de autoridad real o formal, etc. (Le Monde, octubre de 2018).

Ahora es claro que los economistas de la corriente principal se sienten satisfechos por haber cambiado la figura del individuo maximizador por un sujeto que actúa por determinantes sociales complejos y fines también complejos, irreductibles a la maximización de una meta definida por una función objetivo compuesta solo de objetos. Sin embargo, surgen interrogantes sobre tres puntos.

1. Aceptar un homo socialis implica acercarse a Marx, quien en la Introducción de 1857 afirma: "Individuos que producen en sociedad, o sea la producción de los individuos socialmente determinada; este es naturalmente el punto de partida. El cazador o el pescador sueltos y aislados con los que comienzan Smith y Ricardo pertenecen a las imaginaciones desprovistas de fantasía que produjeron las robinsonadas del siglo XVIII". Y también a la figura del individuo que con admiración González encontró en el institucionalista Commons: "La persona tiene un margen de acción importante pero actúa de manera condicionada. Persona institucionalizada, la persona nace en una sociedad en la que ya existen reglas y organizaciones" (p. 77). Si esto se toma en serio es necesaria la ruptura de la ciencia económica con el individualismo metodológico para explicar la economía y la sociedad, y concluir con Benetti y Cartelier: "La noción de individuo, así como la racionalidad que le es atribuida, no se puede definir por fuera de las relaciones en las que él se inserta. Ser un individuo es una realidad social, una forma de existencia de la sociedad. La relación individuo/ sociedad es específica a cada sociedad (p. 19)".

2. Ninguno de los eminentes economistas citados (y González no adopta una posición clara) piensa que el reconocimiento de un individuo cuyo comportamiento tiene determinantes sociales (la consideración de los otros, con creencias y especulaciones sobre lo que ellos hacen, etc.) implica renunciar al modelo básico de la corriente principal, el modelo Arrow-Debreu, y a la microeconomía y la macroeconomía, cuya metodología se reduce a utilizar una idea de comportamiento individual y buscar la determinación de los equilibrios correspondientes. La investigación ha modificado parcialmente un elemento, la hipótesis sobre el individuo, pero ha dejado intacta la idea de equilibrio, el otro concepto central de dicha teoría. Es decir, la aceptación de otra idea del individuo diferente a la del $H E$ no ha 
modificado la matriz de la teoría sino que ha suscitado nuevos modelos de equilibrio, principalmente en microeconomía, en equilibrios parciales y en política microeconómica, que recurren a incentivos, jubilaciones, seguridad social, educación, etc., sin formular un nuevo modelo de equilibrio general con agentes "sociales" o "irracionales" (algo que González apreciaría). En lugar de una ruptura teórica, lo que aparece es el enriquecimiento de una corriente principal centrada en el equilibrio, que valida o justifica modelos con y sin la hipótesis del $H E$, unos más abstractos y otros más "realistas", y sigue manteniendo la idea de que los resultados sociales dependen de que entendamos el comportamiento de los individuos ${ }^{7}$. Así lo confirma Thaler:

La buena noticia es que no es necesario tirar por la borda todo lo que sabemos sobre la manera en que funcionan las economías y los mercados. Las teorías que se basan en la suposición de que somos todos "Econo" (los HE de Thaler) no deben descartarse. Todavia pueden servir como punto de partida para modelos más realistas. En ciertas circunstancias, por ejemplo, cuando los problemas son simples o los actores de la economía poseen capacidades altamente especializadas, los modelos con Econos pueden dar una aproximación de lo que sucede en el mundo real. Pero, como se verá, estas situaciones suelen ser menos la regla que la excepción (ibíd.)

Es evidente que los economistas de la corriente principal poseen la convicción de que tienen un saber aceptable sobre el funcionamiento de las economías, el cual es independiente de la hipótesis de racionalidad individual, como si el asunto fuese poner los distintos individuos racionales o pasionales, sociales o naturales, en el interior de un funcionamiento ya prefijado. Es aquí donde la visión del funcionamiento se vuelve crucial. El economista de la corriente principal responde, desde Arrow-Debreu y con la hipótesis de expectativas racionales, que los agentes económicos se relacionan por medio del equilibrio, cuando todos estén de acuerdo y satisfechos. El equilibrio es entonces la regla (como sugiere Commons) que permite situar al individuo en una relación económica comercial con los otros. Por esta vía, la teoría de la corriente principal sigue en el binomio agente económico y equilibrio, pues la idea de equilibrio (salvo el óptimo) no está en contradicción con alguna idea de racionalidad.

3. Si el equilibrio es la mediación económica básica, aquí hay un problema. En esta visión, los agentes económicos con cualquier comportamiento hacen sus proyectos individuales y son llevados a relacionarse de manera centralista pues el acuerdo general es previo a las transacciones efectivas, circunstancia que no permite diferenciar

7 Tampoco parece que la utilización de la teoría de juegos o la "economía experimental" haya cambiado ese panorama. 
entre economía de mercado y economía socialista. Dicho de otra manera, se acepta el individualismo (con cualquier comportamiento) en la formación de las opciones del individuo pero no en el funcionamiento de la economía. Ahora bien, si estamos de acuerdo en que es necesario definir el individuo como ser social y no en un espacio natural, y si este individuo actúa (tiene relaciones) por fuera del equilibrio, cabe preguntar ¿cuáles son entonces "las reglas y organizaciones" (como dice Commons y acepta González) que permiten situar al individuo en una relación económica comercial realmente descentralizada? Si los economistas quieren explicar el mercado y el capitalismo, deben definir las características económicas que hacen que los agentes se comporten como comerciantes, como capitalistas, como asalariados, como rentistas, sin que exista centralización de las opciones individuales. La teoría económica, después de criticar la idea de individuo de la hipótesis $H E$, ahora debería prestar más atención al segundo aspecto, los individuos en los mecanismos de coordinación económica propios de una economía que funciona de manera descentralizada, por fuera de un equilibrio y sin subastador.

\section{SOBRE LA TEORÍA ECONÓMICA Y SU VALIDACIÓN}

1. Keynes es una teoría del desequilibrio (p.91). Esta es una idea tomada de Leijonhufvud, quien interpreta a Keynes en el marco walrasiano. Aquí basta decir que esta posición es posible porque se adopta la hipótesis de precios rígidos. Si hay flexibilidad de precios, esta versión de la economía de Keynes se vuelve un caso particular de la economía walrasiana. Con razón, estas interpretaciones quedaron relegadas y no han sido aceptadas por los post-keynesianos. Otra posición es posible: Keynes es una teoría de equilibrio macroeconómico siempre y cuando no incluyamos el "mercado de trabajo" en ese equilibrio. El equilibrio con empleo pleno es un caso especial de una economía capitalista, y de esta manera Keynes es la “Teoría General” y la macroeconomía neoclásica, el caso particular.

2. González afirma: "Si el costo de conseguir el dinero es cero, el trueque directo sería equivalente a realizar una transacción mediada por el dinero" (p. 162) y "Keynes busca explicar por qué razón las personas prefieren dinero a otros activos... La moneda es un vínculo entre el presente y el futuro... La demanda de dinero es volátil como el comportamiento de las personas" (p. 163). Es verdad que Keynes $\mathrm{y}$ muchos de sus seguidores repiten esta idea, la cual hace del dinero un activo financiero (como en el modelo monetario neoclásico de generaciones traslapadas) y, por tanto, una economía atemporal de 
mercancías no es monetaria sino de trueque, como si este fuese una técnica de transacciones posible. González no se da cuenta de que esto es la consecuencia de no entender el dinero como medio de cambio y unidad de cuenta, algo diferente a ser posible reserva de valor. La aceptación de la posición de Keynes implicaría una teoría de la demanda de dinero ocasionada por las decisiones individuales debidas a motivos especulativos (un mecanismo de coordinación resultante de estas decisiones) y afirmar que debemos aceptar que una economía atemporal, al no tener presuntamente finanzas, sería una economía de trueque, la cual sabemos que no puede funcionar, como sospechaba el admirado Adam Smith en 1776 y fue probado por Starr y Ostroy.

3. "Un modelo se prefiere porque es compatible con los intereses del poder, así que la disputa entre teorías económicas se resuelve en la esfera política” (p. 17). Con esta teoría conspirativa, González generaliza en exceso y cierra fácilmente el debate. Es indudable que la política económica, sobre todo macroeconómica, se decide por poder político, porque está determinada por los intereses de los electores o tiranos, y no por criterios lógicos y de la buena ciencia positiva. Pero la política económica es el arte y no la ciencia económica propiamente dicha y, así no parece que el análisis económico positivo sea impuesto por el poder político. ¿Cuál es el poder político asociado a las teorías positivas de Smith, Marx, Walras, Arrow, Hicks, Keynes y del mismo González, cuando sabemos que fueron o son intelectuales que lucharon contra el poder político de su época? Afortunadamente nuestro autor tiene dudas, puesto que dice coincidir con McCloskey en que "los modelos permanecen por su capacidad retórica y su poder de convicción [pues]... su relato toca fibras que persuaden y convencen" (p. 171). Puede ser, pero falta exponer mejor las razones para que una teoría "persuada" a las potentes fibras de los intelectuales.

4. González plantea que "en el enfoque de Marx, el ser social determina la consciencia... La libertad y la responsabilidad del sujeto quedan subsumidas en los determinantes sociales y económicos... (Por el contrario) para el liberalismo el sujeto está en capacidad de contrarrestar la incidencia del contexto” (p. 76). Así quiere hacer aparecer al marxismo como una teoría donde la sociedad aplasta el sujeto (algo parecido a su idea de que el equilibrio general walrasiano convierte el sujeto en un animal) para contrastarla con el liberalismo, donde el individuo sería independiente de los determinantes sociales. En realidad, Marx muestra varias veces que es necesario considerar las dos puntas del análisis, la determinación social de los individuos y la autonomía del individuo como ser que tiene un espacio de li- 
bertad y decisión, sobre todo cuando se trata de elaborar la teoría de la economía moderna, caracterizada por la iniciativa privada de los productores ${ }^{8}$. Citemos:

E1 intercambiante ha producido mercancías y precisamente para productores de mercancías. Esto implica: por una parte, que ha producido mercancías como individuo privado independiente, por propia iniciativa, determinado por su propia necesidad y sus capacidades propias, por sî mismo y para sí mismo... Por otra parte, empero, ha producido valor de cambio, un producto que solo se convierte en producto para sí mismo luego de pasar por determinado proceso social... La independencia de la producción individual se complementa de esta suerte con una dependencia social, que encuentra su correspondiente expresión en la división del trabajo (Marx, 1976, p. 167).

En vez de negar por prejuicio el espacio de lo privado en Marx, es necesario reconocer que la dualidad entre lo privado y lo social en el mundo comercial es un mérito del enfoque de Marx, y que precisamente es la que resuelve con una teoría de las relaciones económicas por medio del dinero, y no con la idea de un equilibrio económico. Citemos de nuevo:

Por ende, cada individuo trabaja para sí y su producto no representa nada para sus propios fines, debe realizar intercambios, no solo para participar en el patrimonio productivo general, sino para transformar el propio producto en un medio de vida para sí mismo [...] Es cierto que el cambio, en cuanto está mediado por el valor de cambio y por el dinero, presupone la dependencia reciproca universal de los productos, pero presupone al mismo tiempo el aislamiento completo de sus intereses privados y una división del trabajo social, cuya unidad e integración recíproca existen, por así decirlo, como una necesidad natural externa a los individuos e independiente de ellos. Es la presión sucesiva de la oferta y demanda generales la que media la conexión entre los individuos recíprocamente indiferentes (Marx, 1976 p. 85).

\section{REFERENCIAS BIBLIOGRÁFICAS}

Benetti, C. y Cartelier, J. (1994). L'intérêt limité de l'hypothèse de rationalité individuelle. Cahiers d'Economie Politique, 24-25, 19-35.

Durán, J. D. y Lozano, F. (2018). Sobre la relevancia de los modelos económicos teóricos. Cuadernos de Economía, 37(73), 255-277.

${ }^{8}$ Sin embargo, reconocemos que muchas veces el mismo Marx y los marxistas no reconocen la distancia entre el sujeto y la sociedad. En este error caen, por ejemplo, los que creen que la teoría marxista del valor consiste en afirmar que todo trabajo productor de mercancías es creador de valor, borrando así la diferencia entre trabajo privado y social, dualidad que el autor de El Capital consideraba el secreto para entender la economía moderna. 
Marx, K. (1976). Elementos de la crítica de la economía politica, tomo III. Buenos Aires: Siglo XXI.

Rebeyrol, A. (1999). La pensée economique de Walras. París, Económica. Thaler, R. (2018, 4 de octubre). Nous avons besoin, en économie, d'une approche enrichie qui reconnaisse l'existence des humains. Le Monde, Tirole, J. (2018, 5 de octubre). L’homo economicus a vécu, Le Monde. 